\title{
Tactile Feedback for Artery Detection in Minimally Invasive Robotic Surgery - Preliminary Results of a New Approach
}

\author{
B. Kuebler ${ }^{1}$, R. Gruber ${ }^{1}$, Ch. Joppek ${ }^{2}$, J. Port ${ }^{2}$, G. Passig ${ }^{1}$, J.H. Nagel ${ }^{2}$, and G. Hirzinger ${ }^{1}$ \\ ${ }^{1}$ Institute of Robotics and Mechatronics, German Aerospace Center (DLR), Wessling/Oberpfaffenhofen, Germany \\ ${ }^{2}$ Department of Biomedical Engineering, University of Stuttgart, Germany
}

\begin{abstract}
Minimally invasive robotic surgery (MIRS) entails total absence of haptic feedback due to the spatial separation of patient and surgeon. In conventional surgery, however, palpation to detect superficial arteries by a slight pulsation is an important, commonly applied, and security-relevant procedure. Therefore, an ultrasound based unidirectional sensor for MIRS was developed feeding back kinesthetic impulses to the surgeon-sided haptic input device.
\end{abstract}

Keywords - Minimally Invasive Robotic Surgery, Telesurgery, Ultrasound, Tactility, Modality Substitution.

\section{INTRODUCTION}

Minimally invasive surgery (MIS) is a well-established operation technique, especially for standard procedures such as cholecystectomies (gall bladder removals). Long, slender instruments providing only one degree of freedom (DoF) are guided through small incisions in the patient's skin to access the intra-corporal operation field. Thereby, the epidermis forms a barrier preventing direct view and manual contact. In addition, dexterity is heavily constricted due to the so-called chopstick-effect (inversion of moving direction) and the two missing DoF inside the patient caused by the invariant point of incision (fulcrum point). Getting used to the handling of MIS, therefore, is very time consuming and in addition the falsified haptic feedback is considered to be a major weakness of this technique.

Minimally invasive robotic surgery (MIRS) can overcome these drawbacks using a teleoperated approach: the surgeon sits in an ergonomic posture at a remote console mastering the surgical instruments guided by a patient sided surgical robot. The entire mechanically decoupled arrangement of surgeon and patient, however, results in a total absence of kinesthetic and tactile feedback.

The formerly disputed necessity of kinesthetic feedback is meanwhile considered to be widely accepted [1-3] and approaches have been presented accordingly [4-7]. Moreover, future development in haptic feedback is expected to focus on tactility. Although a lot of research has been performed [8-11], none of the proposed solutions in tactile feedback have prevailed yet.
Therefore, a new approach is presented, focusing on the intra-operative detection of arteries hidden under covering tissue. The objective is to minimize the risk of an accidental dissection of sub-surficial vessels and, thereby, unintentional arterial bleeding. Conventionally (in open surgery), this is performed by palpation and the observance of pulsations. The system is intended to expand the existing MIRS scenario providing kinesthetic feedback [12-14], an inhouse development by a research group of the German Aerospace Center (DLR). It is based on a new, unidirectional ultrasonic probe (see Sec. II. A) and is driven by internally developed electronics (Sec. II. B). Its measurement data are processed (Sec. II. C) to convert the expected palpable pulsations to soft kinesthetic impulses. These are presented on an existing, purchasable haptic interface (Sec. II. D). Preliminary results of the tactile feedback setup are presented in Sec. III. The publication ends with a short conclusion (Sec. IV.) and perspectives on future work (Sec. V.).

\section{Material And Methods}

\section{A. Ultrasonic Probe}

The ultrasonic probe was developed in cooperation with
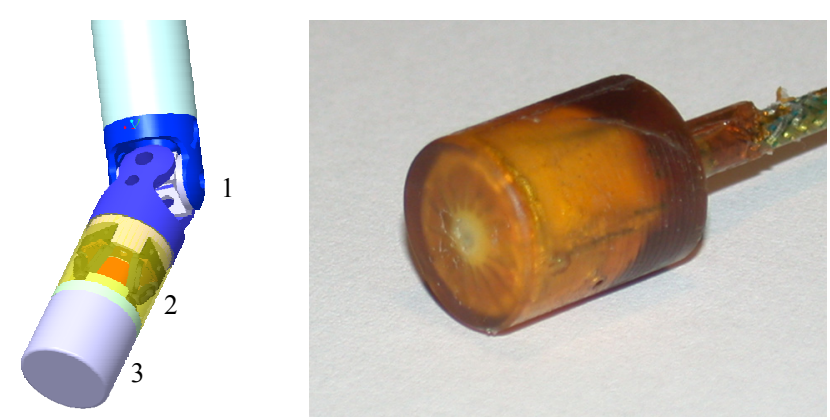

Fig. 1 Left: Planned integration in the existing DLR MIRS instruments; 1) universal joint, 2) 6 DoF force/torque sensor, 3) ultrasonic probe. Right: First Prototype of the unidirectional ultrasonic probe $(\varnothing 10 \mathrm{~mm})$.

the Fraunhofer Institute for Biomedical Engineering (FhGIBMT), St. Ingbert, Germany, and is designed to fit into the 
existing DLR MIRS instruments (see Fig. 1 and [6,7]). Development objective was a probe for reliable detection of vessels beneath covering tissue comparable to the perception of a human finger where a slight pulsation indicates a vessel. Moreover, this pulsation derives from a pulsatile perfusion of the vessel, which can usually also be detected by Doppler sonography. However, conventional Doppler probes are only sensitive to motions in a specific direction. Therefore, the novel unidirectional Doppler probe contains 16 circularly arranged transducer elements (see Fig. 2), since intra-corporal rotational movement of angled instruments with conventional Doppler probes is often cumbersome in MIRS. The transducer elements can all be used either as transmitters or as receivers. This allows the formation of geometrically arbitrary Doppler transmitter/receiver pairs to reliably detect vessels in any planar orientation. The characteristics of the sensor were designed to achieve a penetration depth of 3-4 $\mathrm{mm}$ since deeper vessels would not be palpable manually either. Additionally, vessels under covering tissue deeper than $4 \mathrm{~mm}$ usually do not lie in the primary cutting region of a surgeon since conventionally multiple small and cautious cuts are performed rather than one deep cut. Furthermore, the small penetration depth is advantageous in detecting coronary arteries on the beating heart since disturbances due to ventricular blood turbulences can be avoided. Therefore, a transducer resonance frequency of $20 \mathrm{MHz}$ was chosen, also allowing higher pulse repetition frequencies (PRF). The transducer elements are sealed with a synthetic resin (see Fig. 1) building an adequate impedance changeover to human soft tissue, insulating the transducer to the patient, protecting the assembly, and providing a constant contact geometry.

\section{B. Control Electronics}

For preliminary test purposes a control electronics for only one single transducer element was designed and realized in close cooperation with the University of Stuttgart, Germany, Department of Biomedical Engineering (IBMT Stuttgart). In previous evaluation tests of the transducer probe continuous wave $(\mathrm{cw})$ operation did not prove effective since the frequency of transmitting elements was heavily overlaying measurement signals, probably due to reflections within the fore sealing or the collective central transducer element carrier. Hence, the circuitry was designed to operate the connected transducer element in pulsed wave (pw) mode, using one element as transmitter and receiver in time multiplex. The short signal run-time (penetration depth: 3-4 $\mathrm{mm}$ ) requires relatively fast switching between transmitting and receiving. Additionally, due to the dimensions of the ultrasound elements, only little energy can be coupled into the tissue which makes high transmitter pulse energy and low noise amplification of the small measurement signals necessary.
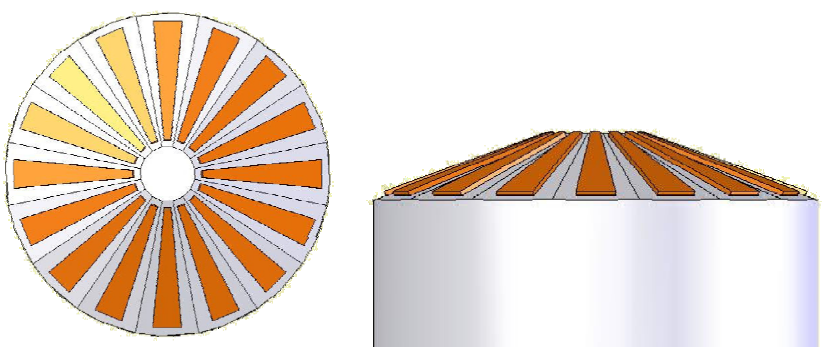

Fig. 2 Circular arrangement of ultrasound transducer elements within the ultrasound probe (CAD drawing). Outer diameter after sealing: $10 \mathrm{~mm}$.

Moreover, the later operation of all transducer elements by using a multiplexer was continually considered during the design process.

\section{Data Acquisition, Signal Processing}

With a previous test setup typical Doppler ultrasound signals of a test person's Arteria radialis (forearm artery) were acquired, digitized, and stored on a conventional personal computer (PC). Subsequently, the data were processed offline with MATLAB. Thereby, the data were first filtered with a standard filter implemented in MATLAB and normalized. Then, a standard peak detection was performed as a first signal analysis. The result (see Fig. 3) was taken as a basis for a signal generation for kinesthetic feedback on an existing haptic feedback interface (see Sec. II. D).

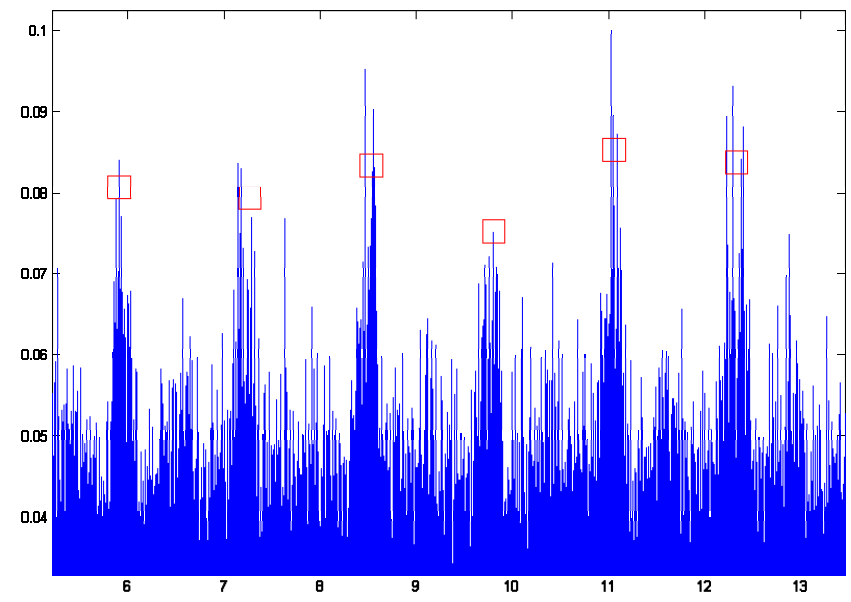

Fig. 3 Preliminary test of a MATLAB based peak detection with a previous transducer setup. Abscissa: time [s]; ordinate: normalized amplitudes. 
In this first attempt, the peaks found in the data stream were weighted equally and submitted to the haptic interface only as uniform impulses. In a later version, the impulses might be fed back more strongly or weakly depending on the ultrasound signal's shape. The signal processing, and therefore the generation of kinesthetic impulses, is presently still offline.

\section{Haptic Interface}

To feed back the measured and computed data in form of short deviated impulses a commercial haptic interface is used (omega.7, see Fig. 4). The system can be run under common software platforms via universal serial bus (USB) and provides 3 passive (rotational) degrees of freedom (DoF) and 4 active (translational and functional) DoF for one hand. In the presented application only the functional DoF is used, the remaining DoF are used to position and orient the instrument tip (i.e. ultrasound probe). The workspace of the functional DoF covers about $25 \mathrm{~mm}$ at achievable continuous forces of $\pm 8.0 \mathrm{~N}$.

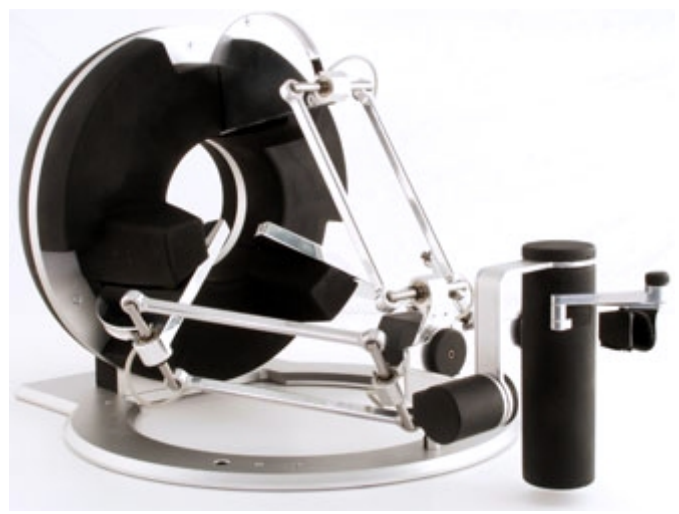

Fig. 4 Haptic interface “omega.7” by ForceDimension, Inc., Lausanne, Switzerland. The system provides 3 passive (rotational) and 4 active (translational and functional) DoF. Photo: ForceDimension [15].

Both powers are not fully used for the standardized soft impulse representing vessel pulsation. Presently, the generated impulses are still offline. However, correlation with artery palpation is conceivable.

\section{RESUlts}

Initially, measurements with the above described electronics and ultrasound probe were performed in a test-bed environment (see Fig. 5) at the IBMT Stuttgart. A water filled plexiglass cylinder $(190 \mathrm{~mm}$ in height to avoid reflections from the bottom) with an arterial phantom (plexiglass tube, inner diameter: $3.5 \mathrm{~mm}$ ) was used. The artery phantom was pulsatively perfused with a blood substitute material to simulate the pulse wave. With these in vitro tests reproducible measurements could be performed to optimize the settings of the electronics.

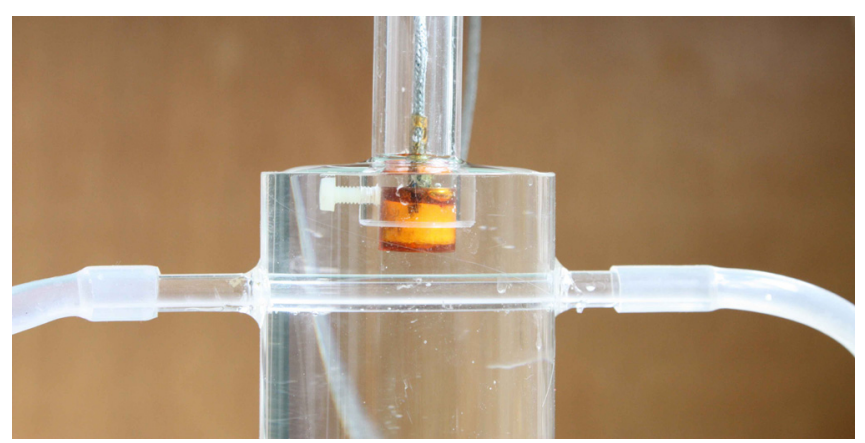

Fig. 5 Test-bed to evaluate the first transducer probe prototype. Ultrasound coupling medium is water; pulsatile fluid flow in an artery phantom.

To prove the above presumptions in vivo, subsequently, a proband's palpable A. radialis was examined. Since the electronics presently only operates one single transducer element the probe had to be adjusted along the flow direction manually. Though clearly audible, the characteristic Doppler sound could not be made visible in the amplitude exposition. However, the spectral Doppler display could be calculated by applying the Fast Fourier Transformation (FFT) to consecutive time windows of 256 data samples (see Fig. 6). Irrelevant frequencies due to physiological hemodynamics (outside $200 \mathrm{~Hz}$ and $5 \mathrm{kHz}$ ) are not shown in Fig. 6. However, the curve progression in Fig. 6 shows characteristics comparable to the typical radialis blood flow.

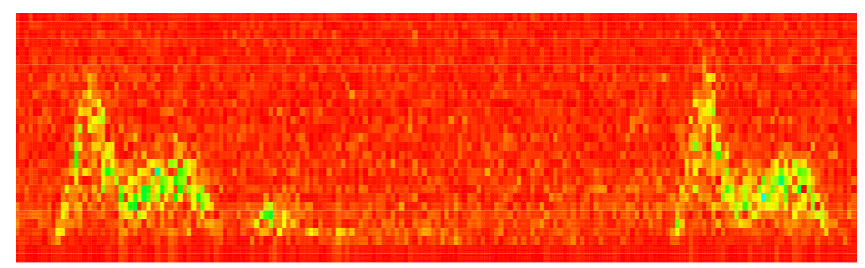

Fig. 6 Calculated spectral Doppler display of a proband's A. radialis; abscissa: time, ordinate: Doppler frequency shift.

With the results shown in Fig. 6 the functionality and basically correct adjustment of the electronics to the transducer elements in the novel ultrasound probe could be demonstrated. Switching time between transmitter and receiver mode is in a suitable range. However, the signal strength could be improved by using a more powerful output amplifier with a faster ramp response since the full capacity of the 
transducer elements is not yet utilized. Moreover, more potential can be realized with a less prototypic, electromagnetically compatible, low-noise assembly.

\section{Conclusions}

The present results of a new tactile feedback system for artery detection in MIRS based on a novel unidirectional ultrasound probe justify further work on the research project.

The presumptions concerning a new transducer for teletactility substitution in a single, closely specified task in MIRS seem to be correct since the A. radialis can be detected and is also palpable. Unidirectionality may be expected due to the measurements, but could not be proven yet since the present evaluation electronics only operates one single transducer element.

The modality substitution and kinesthetic feedback of palpable, hidden, sub-surficial vessels by a soft impulse of a haptic input device's functional DoF (i.e. tactilely expected perception, ultrasound based detection, and kinesthetic feedback) seem feasible and feel satisfactory on the haptic interface. However, online data processing is not yet implemented and a substantiated evaluation (performed by occupational psychologists) of the feedback impression is pending.

\section{Perspectives}

Next development step will be electronic circuits operating all transducer elements in arbitrary activation. Also, the expansion of the evaluated analog front end electronics with a multiplexer, an FPGA based preprocessing unit, and an Ethernet communication interface to a standard $\mathrm{PC}$ is planned.

Online data processing is scheduled to be programmed with RT-Lab (MATLAB tool) and performed on a QNX based PC. The results can then be forwarded to the omega.7 since QNX drivers are available for the hardware.

The next step after functional evaluation of the electronics will be the mechanical integration of the ultrasound probe into the DLR instruments. The force torque sensor presently implemented in the DLR instruments can be integrated to additionally measure contact reactions of the probe with the surrounding tissue.

To evaluate the feedback effect occupational psychological tests by the Human Factors Institute (IfA) of the University of the Bundeswehr Munich were agreed on. In addition, multiple feedback modes (e.g. Doppler sound presentation, flashing lights in the endoscope's video stream or further modes) are planned to be evaluated in these tests.

\section{REFERENCES}

1. Tholey G, Desai JP, Castellanos AE (2005) Force feedback plays a significant role in minimally invasive surgery: results and analysis. Annals of Surgery 241(1):102-9

2. Wagner CR, Howe RD (2005) Mechanisms of performance enhancement with force feedback. Proc EuroHaptics, Pisa, Italy, 2005, pp 21-9

3. Tavakoli M, Aziminejad A, Patel RV, Moallem A (2006) Tool/tissue interaction feedback modalities in robot-assisted lump localization. Proc Int Conf Eng Med Biol Soc, New York, USA, 2006, pp 3854-7

4. Kitagawa M, Dokko D, Okamura AM, Yuh DD (2005) Effect of sensory substitution on suture-manipulation forces for robotic surgical systems. J Thor Cardiovasc Surg 129(1):151-8

5. Mayer H, Nagy I, Knoll A, Braun EU, Bauernschmitt R, Lange R (2007) Haptic feedback in a telepresence system for endoscopic heart surgery. Presence 16(5):459-70 DOI: 10.1162/pres.16.5.459

6. Seibold U, Kuebler B, Hirzinger G (2008) Prototypic force feedback instrument for minimally invasive robotic surgery. In: Bozovic $\mathrm{V}$ (Ed.): Medical Robotics. I-Tech Education and Publishing, pp. $377-$ 400, Vienna, Austria at http://books.i-techonline.com/book.php?id=22

7. Kuebler B, Seibold U, Hirzinger G (2005) Development of actuated and sensor integrated forceps for minimally invasive robotic surgery. Int J Med Rob Comp As Surg 1(3):96-107 DOI: 10.1002/rcs.33

8. Fischer H, Trapp R, Schuele L, Hoffmann B (1997) Actuator array for use in minimally invasive surgery. J de Phys, IV 07(C5):609-14

9. Wagner CR, Lederman SJ, Howe RD (2004) Design and performance of a tactile shape display using RC servomotors. Elec J Hapt Res 3(4) at http://www.haptics-e.org/Vol 03/he-v3n4.pdf

10. Moy G, Wagner C, Fearing RS (2000) A compliant tactile display for teletaction. Proc. vol. 4, Int Conf Rob Autom, San Francisco, USA 2000, pp 3409-15 DOI: 10.1109/ROBOT.2000.845247

11. Franco ML, King CH, Culjat MO, Lewis CE, Bisley JW, Holmes EC, Grundfest WS, Dutson EP (2009) An integrated pneumatic tactile feedback actuator array for robotic surgery. Int J Med Rob Comp As Surg 5(1):13-9 DOI: 10.1002/rcs.224

12. Hagn U, Nickl M, Joerg S, Passig G, Bahls T, Nothhelfer A, Hacker F, Le-Tien L, Albu-Schaeffer A, Konietschke R, Grebenstein M, Warpup R, Haslinger R, Frommberger M, Hirzinger G (2008) The DLR MIRO: a versatile lightweight robot for surgical applications. Ind Rob: Int J 35(4):324-336 DOI: 10.1108/01439910810876427

13. Hagn U, Nickl M, Joerg S, Tobergte A, Kuebler B, Passig G, Groeger M, Froehlich F, Seibold U, Konietschke R, Le-Tien L, AlbuSchaeffer A, Grebenstein M, Ortmaier T, Hirzinger G (2008) DLR MIROSURGE - towards versatility in surgical robotics. Proc curac.08, Leipzig, Germany, pp 143-146

14. Hagn U, Ortmaier T, Konietschke R, Kuebler B, Seibold U, Tobergte A, Nickl M, Joerg S, Hirzinger G (2008) Telemanipulator for Remote Minimally Invasive Surgery. IEEE Rob \& Aut M 15(4):28-38

15. Force Dimension, Inc. at http://www.forcedimension.com 